Research Paper

\title{
Increased resting-state functional connectivity in suprasellar tumor patients with postoperative visual improvement
}

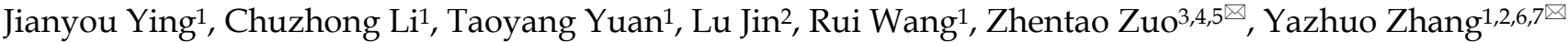 \\ 1. Beijing Neurosurgical Institute, Capital Medical University, Beijing, China \\ 2. Department of Neurosurgery, Beijing Tiantan Hospital, Capital Medical University, Beijing, China \\ 3. State Key Laboratory of Brain and Cognitive Science, Institute of Biophysics, Chinese Academy of Sciences, Beijing, China \\ 4. Sino-Danish College, University of Chinese Academy of Sciences, Beijing, China \\ 5. CAS Center for Excellence in Brain Science and Intelligence Technology, Chinese Academy of Sciences, Beijing, China \\ 6. Beijing Institute for Brain Disorders Brain Tumor Center, Beijing, China \\ 7. China National Clinical Research Center for Neurological Diseases, Beijing, China \\ $\bowtie$ Corresponding author: Zhentao Zuo, ztzuo@bcslab.ibp.ac.cn Yazhuo Zhang, zyz2004520@yeah.net
}

(c) The author(s). This is an open access article distributed under the terms of the Creative Commons Attribution License (https://creativecommons.org/licenses/by/4.0/). See http://ivyspring.com/terms for full terms and conditions.

Received: 2019.04.11; Accepted: 2019.07.10; Published: 2019.08.14

\begin{abstract}
Background and Objective: Large suprasellar tumors often compress the optic chiasm and give rise to visual impairment. Most patients have significantly improved visual function at 1 to 4 months after chiasmal decompression surgery, and only a few individuals regain normal vision at 1 week after surgery. How the recovery of visual function in these patients affects the visual cortex is not fully understood. In this study, we aimed to investigate alterations in brain functional connectivity (FC) in suprasellar tumor patients with visual improvement using resting-state functional magnetic resonance imaging ( $\mathrm{rs}-\mathrm{fMRI})$.

Methods: This longitudinal study was conducted on 13 suprasellar tumor patients who had ophthalmological examinations and rs-fMRI at the following time points: within 1-week preoperation (Pre-op), 1-week postoperation (Post-1w) and 1-month postoperation (Post-1m). The visual impairment score (VIS), local functional correlation (LCOR) and FC values were subjected to one-way ANOVA. Pearson correlation coefficients between changes in the LCOR and clinical factors were calculated.

Results: The VIS was significantly decreased at both Post-1w and Post-1m compared to that at Pre-op. Whole-brain analysis of LCOR values showed that the left V1 (primary occipital cortex) was increased significantly at Post- $1 \mathrm{~m}$ compared to that at Pre-op ( $\mathrm{C}<0.05$, FDR corrected). ROI analysis exhibited a significant negative correlation between the LCOR and VIS changes at Post- $1 \mathrm{~m}$ compared to those at Pre-op $(p<0.05, r=-0.60)$. FC analysis within the visual network showed that the $\mathrm{FC}$ strengths were significantly increased between the left $\mathrm{V} 5$ and the left $\mathrm{V} 4$, right $\mathrm{V} 3 \mathrm{a}$, left V3, left V2d, and right V5 at Post-1m compared to those at Pre-op ( $<<0.05$, FDR corrected). Additionally, the FC strengths were significantly increased between the left V5 and the left V1, right orbital-frontal gyrus and left posterior supramarginal gyrus at the whole-brain network level at Post-1m compared to those at Pre-op ( $p<0.05$, FDR corrected).

Conclusions: Postoperative visual improvement can be reflected by the increased FC of the visual cortex at Post-1 $\mathrm{w}$ and Post- $1 \mathrm{~m}$, especially at Post- $1 \mathrm{~m}$. The LCOR value of the left $\mathrm{V} 1$ was associated with improved visual outcomes and may be used to objectively assess early visual recovery after chiasmal decompression at Post-1m.
\end{abstract}

Key words: suprasellar tumor; visual impairment score (VIS); resting-state functional magnetic resonance imaging (rs-fMRI); local functional correlation (LCOR); functional connectivity (FC). 


\section{Introduction}

Large suprasellar tumors often compress the optic chiasm and give rise to the most common manifestations of progressively decreasing visual acuity or bitemporal hemianopsia [1]. Most suprasellar tumor patients exhibit improved vision function after chiasmal decompression by excision of the lesions [2]. Many of these patients have significantly improved visual function at 1 to 4 months after surgery, and only a few individuals regain normal vision at 1 week after surgery [3]. The subjective assessment of visual function with ophthalmological examination requires cooperation of the participant and is susceptible to various factors [4]. Little is known about how the visual cortex is affected by the recovery of visual function after chiasmal decompression in these patients.

Resting-state functional magnetic resonance imaging (rs-fMRI) plays a vital role in exploring functional interactions between spatially distinct brain regions that reflect the spontaneous fluctuations in brain activity associated with intrinsic behavior [5, 6]. The functional integration of brain networks has been evaluated by data-driven functional connectivity (FC) and local functional correlation (LCOR) analysis [7]. FC analysis was used to investigate significant functional alterations of the brain by examining the temporal correlations between different brain regions using different statistical methods [8]. LCOR, defined as the amount of FC between a voxel and an adjacent voxel across the whole brain in a binary network, may indicate that the voxel in question plays an important role in information processing $[9,10]$. Numerous fMRI studies have shown that the visual cortex undergoes FC changes following peripheral damage to the visual system [11-15]. Task-based fMRI and rs-fMRI studies on pituitary adenoma patients have elucidated that neural dysfunction or FC changes in the visual cortex coincide with partial vision impairment due to chiasmal decompression [16-20]. These studies either transversely focus on preoperative neural activity and FC related to visual impairment or longitudinally concentrate on postoperative neural activity changes in the visual cortex. To our knowledge, there has been no longitudinal rs-fMRI study using LCOR and FC in the vision-related cortex to chart the visual recovery after chiasmal decompression in suprasellar tumor patients.

Taking this approach, thirteen suprasellar tumor patients with visual improvement after chiasmal decompression at the 1-week postoperation (Post-1w) and 1-month postoperation (Post- $1 \mathrm{~m}$ ) time points compared to that within 1-week of the preoperation (Pre-op) were recruited. We attempted to longitudinally investigate alterations of the FC and
LCOR of the visual cortex with visual improvement in 13 suprasellar tumor patients using rs-fMRI in the context of chiasmal decompression and to correlate the fMRI findings with the clinical visual statuses of the patients. We hypothesized that the FC within the vision-related cortex would increase with visual improvement after surgery, especially at the Post- $1 \mathrm{~m}$ time point.

\section{Materials and methods}

\section{Participants}

We initially included 52 suprasellar tumor patients who presented with visual impairment due to lesion-induced compression of the optic chiasm from June 2018 to December 2018. Ultimately, thirteen of these patients with complete data (including ophthalmological examinations and rs-fMRI at the Pre-op, Post-1w and Post-1m timepoints) and postoperative visual recovery at Post- $1 \mathrm{w}$ and Post- $1 \mathrm{~m}$ were recruited. No patients received adjuvant radiotherapy during the Post- $1 \mathrm{~m}$ period. Participants were recruited according to the following exclusion criteria: left-handed; visual impairment related to corneal opacity, glaucoma, cataract, fundus lesions, optic neuropathy, myopia $\geq-6.00 \mathrm{D}$, hyperopia $\geq$ $+6.00 \mathrm{D}$ or other ophthalmologic problems confirmed by ophthalmologic examination; history of stroke, cerebral trauma or other intracranial space-occupying lesions; neurological or mental disorders; history of diabetes, coronary artery disease or other severe illness; no addictions to alcohol or heroin; and inability to undergo MRI and neuroophthalmological examinations.

The Institutional Review Board of Beijing Tiantan Hospital affiliated with Capital Medical University approved the procedures used in the present study. Each participant signed a written informed consent form after understanding our research objectives. Our experimental procedures were based on relevant regulations and guidelines.

\section{Visual assessment}

All patients underwent ophthalmological evaluation at 3 time points. An independent ophthalmologist assessed the visual field, measured the visual acuity and performed fundoscopy. The visual acuity and visual field were separately assessed using Snellen's chart and a standardized automated perimeter (Octopus900 Perimetry, Switzerland). We combined visual field and visual acuity measurements to calculate visual impairment scores (VISs) based on guidelines of the German Ophthalmological Society as reported previously [21, 22]. For example, one patient had a visual acuity of 0.2 in the left eye and 0.4 in the right eye together with a 
bitemporal visual field defect. According to the guidelines of the German Ophthalmological Society, visual acuity is represented by the number 35 , and visual field defects are represented by the number 22 . Twenty-two plus thirty-five equals fifty-seven, and this sum of 57 represents the VIS, which ranges from 0 to 100 . Higher VISs indicate worse visual function and vice versa.

\section{Image acquisition}

The experiment was carried out on a 3T Siemens Prisma MRI scanner (Siemens Healthineers, Erlangen, Germany) using a commercial 64-channel head coil at Beijing Neurosurgical Institute. Each individual high-resolution structural MR image was acquired through a three-dimensional sagittal magnetization-prepared rapid acquisition gradient-echo sequence $(224$ slices; TI/TE/TR = $1000 / 2.22 / 2400 \mathrm{~ms}$; flip angle $=8^{\circ} ;$ bandwidth $=220$ $\mathrm{Hz} / \mathrm{px}$; data matrix $=320 \times 300$; field of view $=256 \times$ $240 \mathrm{~mm}^{2}$ with a resolution of $0.8 \mathrm{~mm}$ isotropic voxels). Two expert radiologists examined the possible lesions in the cortex using structural images for all participants as exclusion criteria. The rs-fMR images were obtained with an echo-planar image sequence (72 slices; $\mathrm{TE}=30 \mathrm{~ms}$; $\mathrm{TR}=710 \mathrm{~ms}$; flip angle $=54^{\circ}$; bandwidth $=2358 \mathrm{~Hz} / \mathrm{px}$; data matrix $=106 \times 106$; multiband factor $=8$; field of view $=212 \times 212 \mathrm{~mm}^{2}$ with a resolution of $2.0 \mathrm{~mm}$ isotropic voxels) [23]. During a 7-min and 41-s functional scan, patients were required to relax, not think of anything and gaze at a fixation point in the central screen throughout the session. After the scan, each subject was asked if he/she remained awake during the whole procedure.

\section{Data preprocessing}

The preprocessing of rs-fMRI data was conducted with Statistical Parametric Mapping (SPM12, http://www.fil.ion.ucl.ac.uk/spm) and the CONN toolbox [24]. The first ten volumes were removed to avoid initial signal instability. The preprocessing steps comprised head motion correction, slice timing correction, spatial normalization to the standard Montreal Neurological Institute (MNI) brain space $(2 \mathrm{~mm})$, and spatial smoothing (6 mm full width half maximum Gaussian kernel). To eliminate physiological high-frequency cardiac and respiratory noise and reduce low-frequency drift, a temporal bandpass $(0.01-0.1 \mathrm{~Hz})$ was performed. Linear trend removal within each time series was also carried out. The head motion, global brain signal, white matter signal and cerebrospinal fluid (CSF) signal were regressed out from the time course of rs-fMRI.

\section{LCOR analysis}

LCOR maps characterized local brain coherence by integrating the spatial Pearson correlation function for each voxel. LCOR is characterized by the strength and sign of connectivity between a given voxel and the neighboring areas in the brain. The FC strength was measured based on the Pearson correlation coefficient of the time courses between the current and neighboring voxels. LCOR is defined as the average of correlation coefficients between each individual voxel and a region (kernel size was $6 \mathrm{~mm}$ ) of neighboring voxels [9].

\section{Relationship between LCOR and clinical factors}

Pearson correlation analysis between changes in LCOR values in the vision-related cortex and clinical factors (VIS and duration) was performed to determine whether the LCOR varied with clinical factors. Significance was determined using $\mathrm{p}<0.05$.

Table 1. MNI coordinates of ROI-wise seed nodes.

\begin{tabular}{llll}
\hline Region name & \multicolumn{2}{l}{ Peak MNI coordinate } \\
\cline { 2 - 4 } & $\mathrm{x}$ & $\mathrm{y}$ & $\mathrm{z}$ \\
\hline V1.L & -6 & -87 & 2 \\
V2v.L & -7 & -79 & -5 \\
V2d.L & -11 & -96 & 8 \\
VP.L & -14 & -76 & -8 \\
V3.L & -18 & -93 & 12 \\
V3a.L & -26 & -87 & 17 \\
V4.L & -22 & -70 & -8 \\
V5.L & -41 & -75 & 3 \\
V1.R & 8 & -83 & 5 \\
V2v.R & 10 & -74 & -1 \\
V2d.R & 10 & -91 & 11 \\
VP.R & 15 & -73 & 6 \\
V3.R & 15 & -91 & 16 \\
V3a.R & 20 & -86 & 21 \\
V4.R & 22 & -77 & -11 \\
V5.R & 46 & -66 & -1 \\
\hline
\end{tabular}

L: left; R: right.

\section{Seed-based resting-state functional connectivity (RSFC) analysis}

To calculate the RSFC, we used ROIs as seeds to assess correlations between adjacent brain regions. In this study, 16 nodes (including 8 nodes in each brain hemisphere, V1, V2v, V2d, V3a, V3, VP, V4, and V5; their MNI coordinates, see Table 1) with a $5 \mathrm{~mm}$ radius were defined as ROIs to calculate the ROI-wise correlation matrix in the visual networks [25]. The coordinates of these selected nodes were acquired according to a previous study [26]. First, all clusters were extracted from the corrected correlation map in the standard MNI space. Then, we calculated the Pearson correlation coefficients between the average time courses for every seed region and then converted 
to $\mathrm{z}$ values by Fisher's $\mathrm{z}$ transformation. Finally, the brain regions with significantly different FC to the seed regions were confirmed by two-sample paired $\mathrm{t}$-tests between the preoperative and Post- $1 \mathrm{~m}$ periods. Significance was determined using $p F D R$ (false discovery rate) $<0.05$ at the cluster level and $p<0.001$ at the voxel level.

\section{Results}

\section{Demographic and clinical factors}

The demographic and clinical features of the suprasellar tumor patients are shown in Table 2. A total of 13 participants were recruited, including 8 $(61.54 \%)$ female patients and $5(38.46 \%)$ male patients. The age ranged from 34 years to 58 years with a mean age of $46.46 \pm 6.86$ years. The duration ranged from 1 month to 36 months with a mean time of $8.47 \pm 10.33$ months. Eleven of the suprasellar tumors were pituitary adenomas, and two were meningiomas. All lesions extended upward to the sella, and 6 also extended to the cavernous sinus. The vision functions of all patients with preoperative partial visual impairment were improved via the transsphenoidal approach except for one, who was treated with the transcranial approach. The VIS decreased at both Post- $1 \mathrm{w}$ and Post- $1 \mathrm{~m}$ in all patients compared to that at Pre-op. One-way ANOVA was performed among the 3 time points, and a significant main effect was observed $(\mathrm{F}(2,24)=48.09, p<0.0001)$. Tukey's multiple comparisons test was then performed. A paired t-test showed significant treatment effects between Pre-op $(25.62 \pm 10.44)$ and Post- $1 \mathrm{w}(12.69 \pm 10.27)(\mathrm{t}(12)=5.14$, $p<0.001)$ and significant recovery effects between Post- $1 \mathrm{w}$ and Post-1m $(4.62 \pm 6.70)(\mathrm{t}(12)=4.68, p<$ 0.002) (see Figure 1).
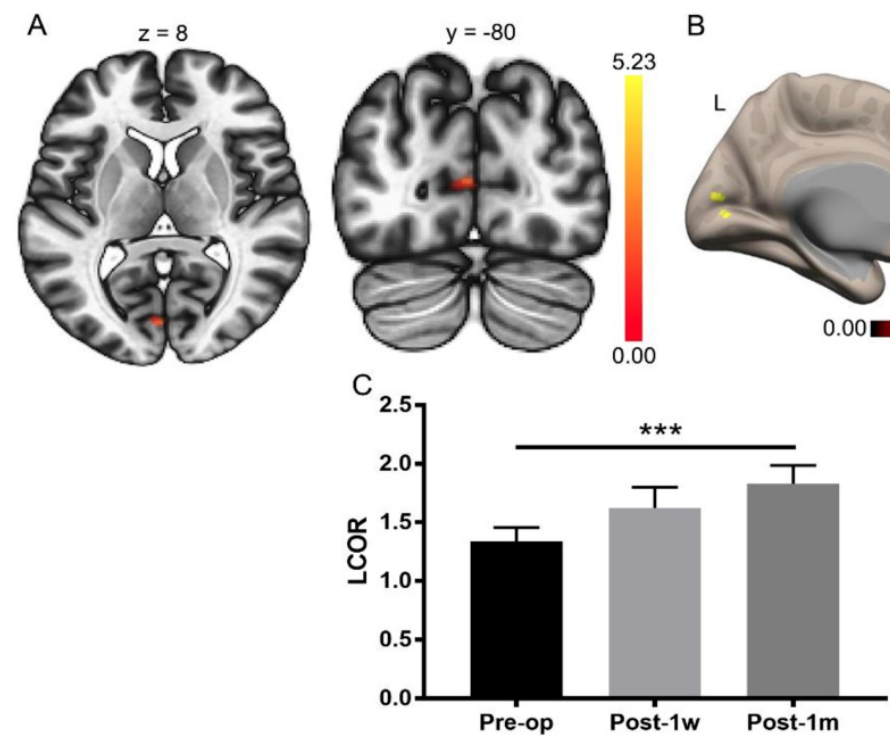

Figure 2. Whole-brain LCOR analysis of Pre-op and Post-1m. (A) Statistical parametric map (axial and coronal view). (B) Surface brains (3D view). (C) The LCOR values of the left $\mathrm{V} 1$ at Post- $1 \mathrm{~m}$ are significantly higher than those at Pre-op $(t(12)=-6.07, p<0.001)(* * *)<0.001)$. L, left; $R$, right.

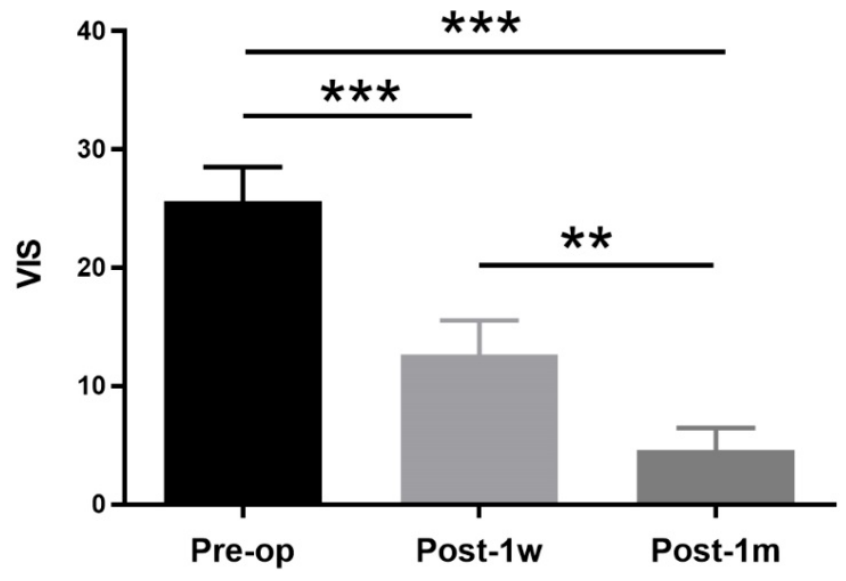

Figure 1. VISs at Pre-op, Post-1w and Post-1m. The VIS gradually decreased after surgery. A significant treatment effect was observed from Pre-op to Post-Iw $(\mathrm{t}(12)=5.14, \mathrm{p}<0.001)$, and a significant recovery effect was observed from Post-1w to Post-1m $(t(12)=4.68, p<0.002)(* * p<0.01$, **** $p<0.001)$.

\section{Local functional correlation analysis}

The visual function improved substantially after Post-1m, as previously reported [3]. A paired t-test was applied to the whole-brain LCOR analysis of Pre-op and Post-1m with a cluster-level threshold of $p$ FDR $<0.05$ and a voxel-level threshold of $p<0.001$. The only significant difference was found in the left V1 (primary visual cortex) with center MNI coordinates $(-2,-82,10)$, and the cluster size was 44 voxels. The LCOR values were extracted from the left V1 across the Pre-op, Post-1w, and Post-1m time points (see Table 3). One-way ANOVA was performed among the Pre-op, Post- $1 \mathrm{w}$, and Post- $1 \mathrm{~m}$ groups, and the main effect was significant $(\mathrm{F}(2,24)=$ $9.15, p<0.005)$. Tukey's multiple comparisons test was then performed, revealing significantly higher LCOR values in the left V1 at Post-1m than at Pre-op (see Table 3 and Figure 2). There were no significant differences between the other groups.

B

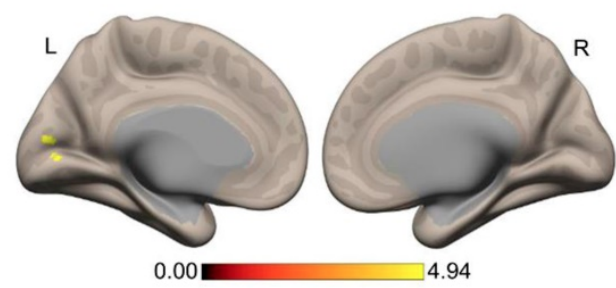


Table 2. Demographic data and clinical factors of all patients.

\begin{tabular}{|c|c|c|c|c|c|c|c|c|c|c|}
\hline & \multirow[t]{2}{*}{$\mathrm{n}$} & \multirow[t]{2}{*}{ Gender } & \multirow[t]{2}{*}{ Age (years) } & \multirow{2}{*}{$\begin{array}{l}\text { Duration } \\
\text { (months) }\end{array}$} & \multirow[t]{2}{*}{ Handedness } & \multirow[t]{2}{*}{ Approach } & \multirow[t]{2}{*}{ Pathology } & \multicolumn{3}{|l|}{ VIS } \\
\hline & & & & & & & & Pre-op & Post-1w & Post-1m \\
\hline Patients & 13 & $8 \mathrm{~F} / 5 \mathrm{M}$ & $46.5 \pm 6.9$ & $8.5 \pm 10.3$ & right & 12TS / 1TC & 11PA / 2ME & $25.6 \pm 10.4$ & $12.5 \pm 9.8$ & $4.6 \pm 6.7$ \\
\hline
\end{tabular}

F: female; M: male; TS: transsphenoidal; TC: transcranial; PA: pituitary adenoma; ME: meningioma.

Table 3. LCOR values in the left V1 at the Pre-op, Post- $1 \mathrm{w}$ and Post- $1 \mathrm{~m}$ time points.

\begin{tabular}{llllllll}
\hline Region & Mean (SD) & & $\mathrm{F}(2,24) / \mathrm{p}$ & $\mathrm{t}(12) / \mathrm{p}$ & & \\
\cline { 2 - 8 } & Pre-op & Post-1w & Post-1m & one-way ANOVA & Pre-op vs Post-1m & Pre-op vs Post-1w & Post-1w vs Post-1m \\
\hline Left V1 & $1.34(0.43)$ & $1.62(0.64)$ & $1.83(0.57)$ & $9.15 /<0.005$ & $-6.07 /<0.0002$ & $-2.15 / 0.121$ & $-1.64 / 0.265$ \\
\hline
\end{tabular}

\section{Correlation analysis}

Correlation analysis of changes in LCOR values in the vision-related cortex and clinical factors (VIS and duration) was performed at the 3 time points, revealing that the increased LCOR values in the left V1 were associated with decreased VISs after surgery. The changes in LCOR values in the left V1 and VIS from the Pre-op period to the Post- $1 \mathrm{~m}$ period were significantly negatively correlated $(r=-0.60,-0.8652$ to $-0.07405, p<0.05$, see Figure 3 ). No other significant correlations were observed.

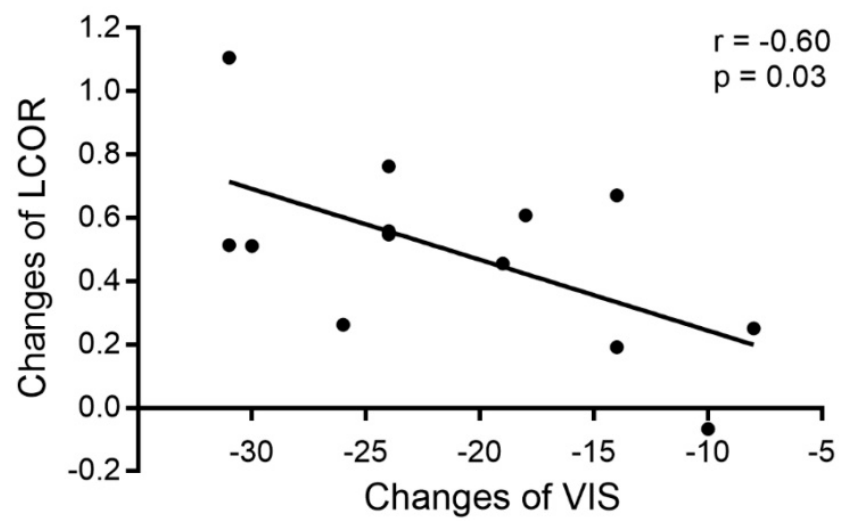

Figure 3. Correlation between changes in LCOR values in the left $\mathrm{V} 1$ and changes in VIS from the Pre-op period to the Post- $1 \mathrm{~m}$ period.

\section{Visual network analysis}

ROI-to-ROI Pearson correlation analyses within the visual network across a time series were conducted using CONN. Multiple comparisoncorrected paired t-tests were performed between the Pre-op and Post-1m groups. The correlation coefficients of FC significantly increased between the left V5 and the left V2d, right V3a, left V3, left V4 and right V5 (see Table 4 and Figure 4A).

Table 4. Visual network with significantly increased FC between the Post- $1 \mathrm{~m}$ and Pre-op time points.

\begin{tabular}{llll}
\hline Network & \multicolumn{2}{c}{ T } & $p$ FDR \\
\cline { 1 - 2 } seed & target & & \\
\hline V5.L & V2d.L & 3.68 & $<0.05$ \\
V5.L & V3.L & 3.56 & $<0.05$ \\
V5.L & V3a.R & 3.08 & $<0.05$ \\
V5.L & V4.L & 2.94 & $<0.05$ \\
V5.L & V5.R & 2.84 & $<0.05$ \\
\hline
\end{tabular}

L: left; R: right.

The correlation coefficients from the above five edges with significant changes in FC were extracted, and two-way ANOVA of 3 time points $\times 5$ edges was performed. Significant effects across Pre-op, Post- $1 \mathrm{w}$ and Post- $1 \mathrm{~m}(\mathrm{~F}(2,24)=11.03, p<0.0005)$ and main effects for edges $(\mathrm{F}(4,48)=3.32, p<0.02)$ were observed. However, no significant interaction effect was found $(\mathrm{F}(8,96)=0.82, p=0.59)$.

One-way ANOVAs were performed on each edge. For the FC strength of edges between the left V5 and left V2d, left V3, right V3a, and left V4, significant main effects were observed across the 3 time points (Geisser-Greenhouse corrected, see Table 5). Tukey's multiple comparisons test was then performed. Paired t-tests showed significant effects on the edges of left V5 and left V2d, left V5 and left V3, left V5 and right V3a, left V5 and left V4 and left V5 and right V5 between Pre-op and Post-1m and on the edges of left V5 and right V3a and left V5 and left V4 between Pre-op and Post-1w (see Figure 4B and Table 5).

Table 5. FC strengths of the edges between IV5 and IV2d, IV3, rV3a, IV4 and Rv5 at Pre-op, Post-Iw and Post-1m.

\begin{tabular}{|c|c|c|c|c|c|c|c|}
\hline \multirow[t]{2}{*}{ Edges } & \multicolumn{3}{|l|}{ Mean (SD) } & \multirow{2}{*}{$\begin{array}{l}\mathrm{F}(2,24) / \mathrm{p} \\
\text { one-way ANOVA }\end{array}$} & \multicolumn{3}{|l|}{$t(12) / p$} \\
\hline & Pre-op & Post-1w & Post-1m & & Pre-op vs Post-1m & Pre-op vs Post-1w & Post-1w vs Post-1m \\
\hline lV5 to lV2d & $0.09(0.17)$ & $0.22(0.22)$ & $0.32(0.15)$ & $5.48 /<0.02$ & $-5.21 /<0.01$ & $-2.58 / 0.20$ & $-1.87 / 0.41$ \\
\hline 1V5 to $1 \mathrm{~V} 3$ & $0.22(0.15)$ & $0.31(0.24)$ & $0.46(0.20)$ & $4.43 /<0.05$ & $-5.04 /<0.01$ & $-1.90 / 0.40$ & $-2.02 / 0.35$ \\
\hline IV5 to $\mathrm{rV3a}$ & $0.19(0.18)$ & $0.43(0.31)$ & $0.38(0.22)$ & $7.11 /<0.002$ & $-4.36 /<0.05$ & $-5.29 /<0.001$ & $1.01 / 0.76$ \\
\hline IV5 to $1 \mathrm{~V} 4$ & $0.18(0.18)$ & $0.38(0.25)$ & $0.36(0.24)$ & $5.11 /<0.02$ & $-4.15 /<0.05$ & $-4.24 /<0.05$ & $0.36 / 0.96$ \\
\hline IV5 to rV5 & $0.33(0.35)$ & $0.50(0.29)$ & $0.52(0.32)$ & $3.03 / 0.08$ & $-5.02 /<0.05$ & $-2.38 / 0.25$ & $-0.29 / 0.97$ \\
\hline
\end{tabular}



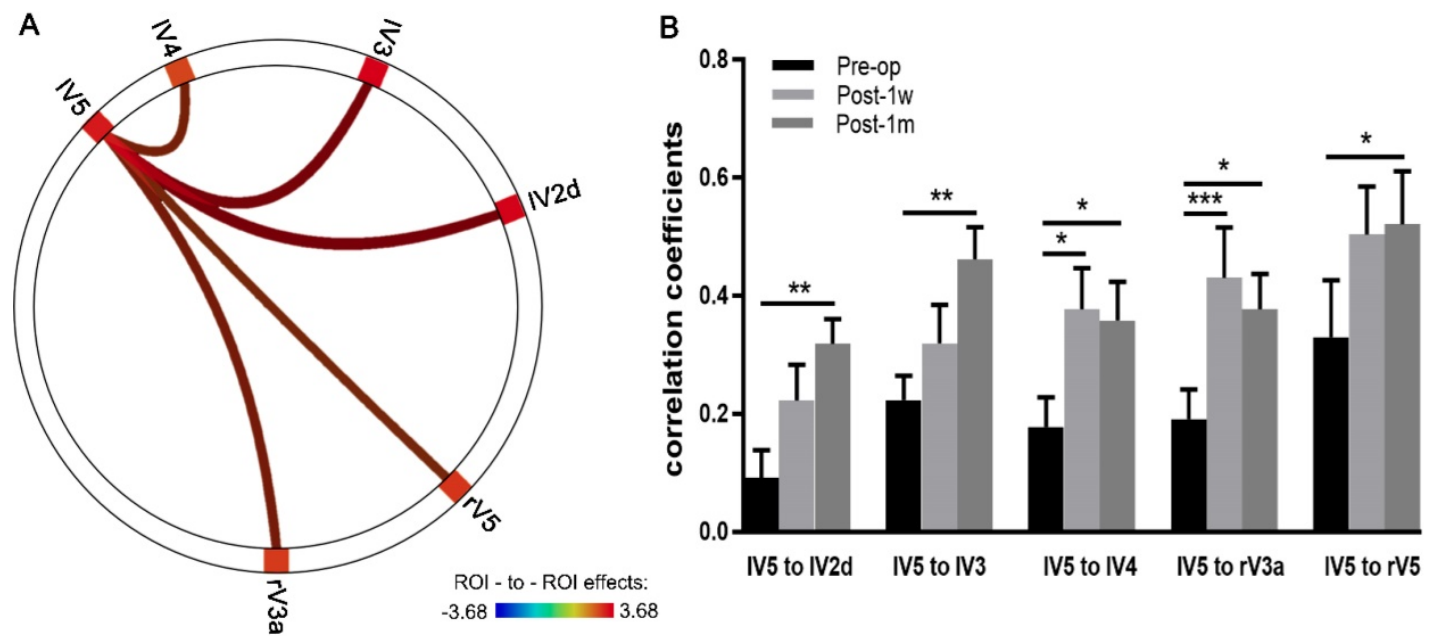

Figure 4. Depictions of FC strength improved in the visual networks after surgery. (A) Circle plots depict the visual network with significantly increased FC between the Pre-op and Post-1m period (the left V5 and the left V2d, right V3a, left V3, left V4 and right V5). (B) Coefficients of five edges at the Pre-op, Post- $1 \mathrm{w}$ and Post-1 m time points $(* \mathrm{p}<0.05, * * \mathrm{p}<0.01$, *** $\mathrm{p}<0.001)$. l, left; $r$, right.

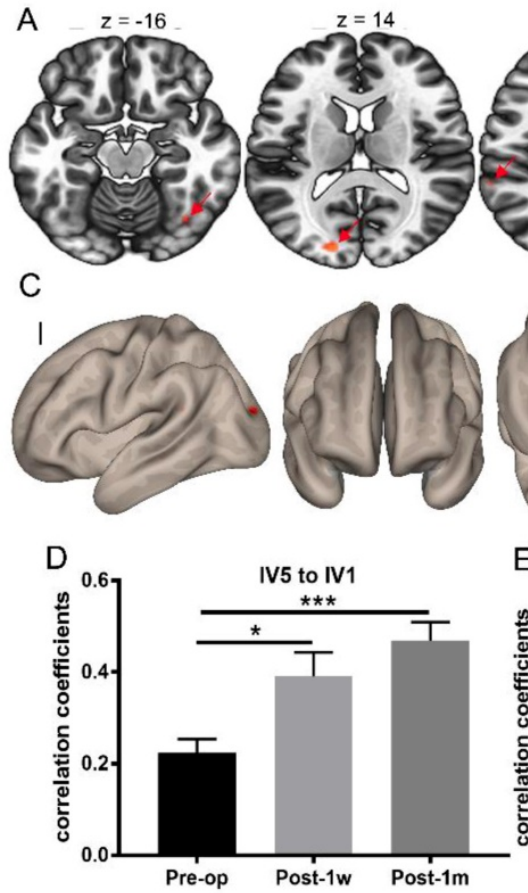

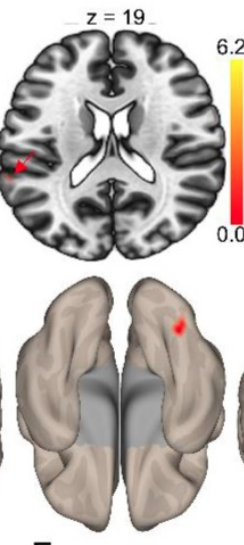

E
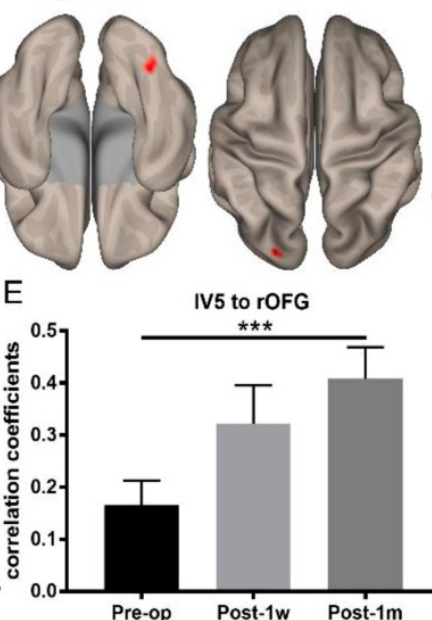
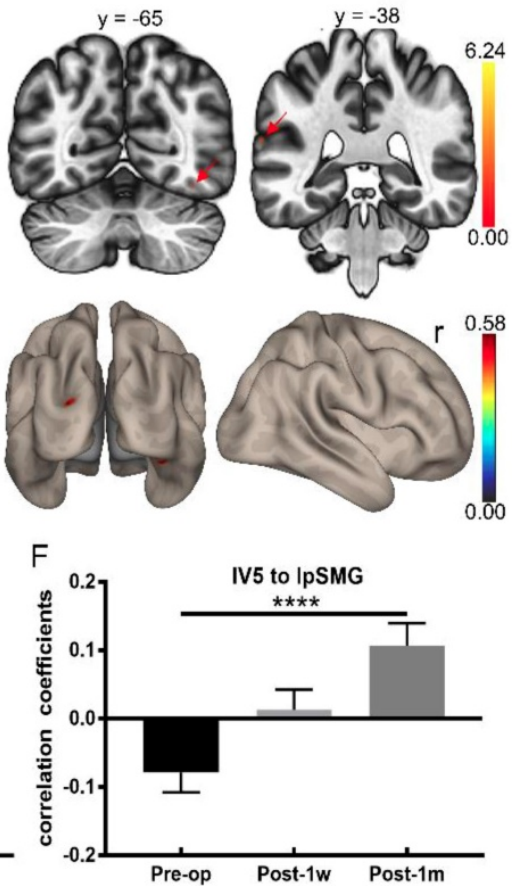

Figure 5. Whole-brain FC strength alterations after surgery determined using the left V5 as a seed. (A) Statistical parametric map between Pre-op and Post-1m (axial view, arrows point to the significantly different regions). (B) Statistical parametric map between Pre-op and Post-1m (coronal view, arrows point to the significantly different regions). (C) Surface brain between Pre-op and Post-1m (3D view). Coefficients between the left V5 and left V1 (D), rOFG (E) and lpSMG (F) at Pre-op, Post-1w and Post- $1 \mathrm{~m}(* \mathrm{p}<0.05$, *** $\mathrm{p}<0.001)$. l: left; r: right.

\section{Whole-brain FC analysis based on left V5.}

Whole-brain FC analysis was conducted using left V5 as the seed. Significant FC changes were observed between Pre-op and Post-1m using $p$ FDR $<$ 0.05 at the cluster level and $p<0.001$ at the voxel level as the thresholds. The FC map of the left V5 was significantly different regarding connectivity with the left V1, right orbital-frontal gyrus and left posterior supramarginal gyrus regions (see Table 6 and Figure $5 \mathrm{~A}-\mathrm{C})$.
Table 6. Differences in whole-brain FC determined using the left V5 as the seed at Pre-op and Post-1m.

\begin{tabular}{llllllll}
\hline Region & \multicolumn{2}{c}{ Coordinate } & \multicolumn{2}{c}{ Cluster size (voxels) } & T & $p$ FDR \\
\cline { 2 - 4 } & $\mathrm{x}$ & $\mathrm{y}$ & $\mathrm{z}$ & & 7.65 & 0.008 \\
\hline Left V1 & -18 & -90 & 14 & 44 & 6.03 & 0.017 \\
rOFG & 36 & -6 & -14 & 34 & 5.75 & 0.042 \\
lpSMG, & -66 & -38 & 20 & 26 & & \\
\hline
\end{tabular}

For FC between the left V5 and left V1 (Geisser-Greenhouse corrected $\mathrm{F}(1.78,21.32)=12.96, p$ $<0.0003$ ), rOFG (Geisser-Greenhouse corrected $\mathrm{F}(1.75,20.97)=10.85, p<0.001)$ and lpSMG 
(Geisser-Greenhouse corrected $\mathrm{F}(1.48,17.82)=13.41, p$ $<0.0007)$, significant main effects were observed. Tukey's multiple comparisons test was then performed. Paired t-tests showed significant treatment effects in the FC of the left V5 and left V1, left V5 and rOFG and left V5 and lpSMG between Pre-op and Post-1m and in the FC of the left V5 and left V1 between Pre-op and Post-1w (see Figure 5D-F and Table 7).

\section{Discussion}

The current study explored FC changes in the vision-related cortex in suprasellar tumor patients with postoperative visual improvement at the voxel level, ROI level and whole-brain network level based on rs-fMRI. The LCOR values significantly increased in the left V1 at Post-1m compared to those at Pre-op, and LCOR changes were negatively correlated with VIS changes from Pre-op to Post- $1 \mathrm{~m}$. The FC strength significantly increased between the left V5 and other brain regions at both the ROI level and the whole-brain network level. The patients with visual improvement after chiasmal decompression exhibited improved FC in the vision-related cortex at Post- $1 \mathrm{w}$ and Post- $1 \mathrm{~m}$, especially at the Post- $1 \mathrm{~m}$.

The regional homogeneity ( $\mathrm{ReHo}$ ) values of the occipital cortex were reduced in pituitary adenoma patients compared with those in healthy controls [20]. In addition, the ReHo values were increased when comparing postoperative pituitary adenoma patients with visual improvement with preoperative patients [19]. However, no direct evidence has shown the recovery effects of suprasellar tumor patients after chiasm decompression. In this study, 13 suprasellar tumor patients with visual improvement after surgery were included, and longitudinal comparisons between the Pre-op and Post-1w and Post-1m time points were conducted. LCOR values from the left V1 were increased after surgery at Post- $1 \mathrm{w}$ and Post-1m compared with those at Pre-op, while the differences were significant at only Post-1m and not at Post-1w. V1 is subjected to internal and external factors and varies with different visual statuses [27]. Pituitary adenoma tumors reduce the signals from the retina into the visual cortex, which may reduce the activity of V1 $[18,20]$ and the local FC within the V1 region, causing blurred images from visual input. Decompression of the optic chiasm allowed more visual stimuli to be received from the retina, as determined based on the observation of increased visual cortex activity and the rebuilding of FC with adjacent neurons. However, the connection was not so stable at Post- $1 \mathrm{w}$, and some patients thus did not gain vision improvement at this time point; these patients might need a longer recovery time $[16,17]$, such as 1-4 months.

Although the VISs of the patients we included in this study were significantly decreased after chiasmal decompression, the LCOR values in the left V1 were significantly higher than those at Pre-op at only Post-1m and not at Post-1w. It seems that the visual function occurred before the visual network recovered. The VIS measures and subjectively assesses the deficit of visual function [21, 22]. After chiasmal decompression, retinal signals transmitted to the visual cortex could increase as the visual field improves. However, the local FC may still be unstable, as some studies have shown that visual acuity improvement lags behind that of the visual field [28-31], while others have shown that improvement of the visual field lags behind that of visual acuity $[32,33]$. One study on pituitary adenoma patients showed that visual function was significantly improved at 1 to 4 months after surgery, and only a few individuals regained normal vision at 1 week after surgery [3]. In this study, we found that changes in the LCOR values in the left V1 lagged behind visual changes, which might mean that changes in the LCOR were caused by increased visual information stimulation. The brain will change its FC to improve the efficiency of information processing so that its function can adapt to the current needs [34-36]. Many fMRI studies have documented the involvement of V1 in visual learning, which can increase neural activity and FC with trained stimuli [37-40].

Hyperconnectivity was a common response to neurological disruption in patients with multiple sclerosis, traumatic brain injury, idiopathic generalized epilepsy, mild cognitive impairment, and Alzheimer's disease [41-44]. Some hyperconnectivity may exist within the visual cortex after surgery in pituitary adenoma patients. Previous studies demonstrated that the ReHo and FC of the V1 region in pituitary adenoma patients were significantly decreased compared to those in the healthy control group $[18,20]$.

Table 7. FC strengths of edges between IV5 and IVI, rOFG and IpSMG at Pre-op, Post-1w and Post-1m.

\begin{tabular}{|c|c|c|c|c|c|c|c|}
\hline \multirow[t]{2}{*}{ Edges } & \multicolumn{3}{|l|}{ Mean (SD) } & \multirow{2}{*}{$\begin{array}{l}\mathrm{F}(2,24) / \mathrm{p} \\
\text { one-way ANOVA }\end{array}$} & \multicolumn{3}{|l|}{$\mathrm{t}(12) / \mathrm{p}$} \\
\hline & Pre-op & Post-1w & Post-1m & & Pre-op vs Post-1m & Pre-op vs Post-1w & Post-1w vs Post-1m \\
\hline lV5 to lV1 & $0.22(0.11)$ & $0.39(0.19)$ & $0.47(0.15)$ & $12.96 /<0.0003$ & $-8.53 /<0.0002$ & $-4.21 /<0.05$ & $-2.24 / 0.29$ \\
\hline IV5 to rOFG & $0.17(0.17)$ & $0.32(0.26)$ & $0.41(0.22)$ & $10.85 /<0.001$ & $-8.133 / 0.0002$ & $-3.71 / 0.054$ & $-2.21 / 0.29$ \\
\hline 1V5 to $1 \mathrm{pSMG}$ & $0.08(0.10)$ & $0.01(0.11)$ & $0.11(0.12)$ & $13.41 /<0.0007$ & $-10.82 /<0.0001$ & $2.98 / 0.13$ & $-3.58 / 0.06$ \\
\hline
\end{tabular}


In our study, the LCOR values in the left V1 gradually increased after chiasmal decompression in the process of visual recovery, and the LCOR values in the left V1 were significantly increased at only the Post- $1 \mathrm{~m}$ compared to those at Pre-op. The LOCR values at Post- $1 \mathrm{~m}$ were not higher than those at Post-1w, and those at Post-1w were not higher than those at Pre-op. Therefore, we believe that the increase in FC strength after surgery was a functional recovery mechanism of the visual cortex through connecting adjacent neurons rather than hyperconnectivity within the visual network. Changes in the LCOR value were correlated with the recovery of vision function, as described by the VIS.

Located at the top of the ascending branch of the middle temporal sulcus, V5 (MT+) is a visual area specialized for motion perception in the visual modality [45, 46]. The left V5 is activated when a moving object is imaged, but that in the right hemisphere is not [47]. In the present study, visual network analysis showed that the positive FC values between the left V5 and the left V2d, left V3, left V4, right V3a and right V5 were increased at Post-1m compared to those at Pre-op in suprasellar tumor patients with visual improvement. When the LCOR values increase, the details of the image or object become sharper, which leads to enhanced sensitivity of motion perception. The left V5 increased the FC with the dorsal stream of the visual cortex, which improved the "where" pathway.

More interestingly, whole-brain FC analysis using left V 5 as the seed revealed that the FC map of the left V5 with the left V1, right orbital-frontal gyrus and left posterior supramarginal gyrus was significantly altered before and after the operation. These regions beyond the visual cortex are involved in the processes of language and sensation [48-50], and the reason for the alterations in FC are unknown. We speculated that these patients might exhibit increased communicative ability with vision recovery after surgery, and the connection among higher-order cognitive networks would then be strengthened, as mentioned earlier in association with the fact that the brain increases its FC to adapt to information processing needs [34-36].

There are some limitations in the present study. First, the small sample sizes constituted the main limitation of this study, which might reduce the reliability of the findings. Second, a control sample was not included in the study, and having a control sample would be beneficial for clarifying FC changes before and after surgery. Third, we explored the FC changes with only rs-fMRI, and other neural changes associated with improved vision might be determinable using voxel-based morphometry
(VBM), diffusion tensor imaging (DTI) and task-based fMRI analyses. Multimodal neuroimaging analysis may be the direction for investigating the mechanisms underlying structural and functional alterations.

\section{Conclusion}

In summary, postoperative visual improvement can be reflected in the increased FC of the visual cortex at Post- $1 \mathrm{w}$ and Post- $1 \mathrm{~m}$, especially at Post- $1 \mathrm{~m}$. The increased FC of the vision-related cortex might indicate that the rehabilitation course changed in accordance with improved vision input. The LCOR of the left V1 was associated with improved visual outcomes and may be used to objectively assess early visual recovery after chiasmal decompression at the Post- $1 \mathrm{~m}$ time point. Our study may provide important insights into functional alterations related to the vision-related cortices of suprasellar tumor patients with visual improvement.

\section{Acknowledgments}

We would like to thank the colleagues at Beijing Tiantan Hospital and Beijing Neurosurgical Institute who assisted with this study and the volunteers who participated in the study.

\section{Author contributions}

JY recruited the participants, collected the clinical data, collected the MRI data, interpreted the data and drafted the manuscript. $\mathrm{ZZ}$ conducted the MRI data analyses, provided advice on data interpretation and reviewed the manuscript. TY and LJ collected the clinical and MRI data. RW collected the MRI data. CL and YZ designed the framework of this study and provided advice on interpreting the data.

\section{Funding}

This work was supported in part by the Ministry of Science and Technology of China (2015CB351701), Beijing Municipal Science \& Technology Commission (Z171100000117002), National Nature Science Foundation of China grant (91132302, 31730039 and 31671133), National Major Scientific Instruments and Equipment Development Project (ZDYZ2015-2) and Chinese Academy of Sciences Strategic Priority Research Program B grants (XDBS32000000).

\section{Competing Interests}

The authors declare that the research was conducted in the absence of any commercial or financial relationships that could be construed as a potential conflict of interest. 


\section{References}

1. Hajiabadi M, Samii M, Fahlbusch R. A preliminary study of the clinical application of optic pathway diffusion tensor tractography in suprasellar tumor surgery: preoperative, intraoperative, and postoperative assessment. Journal of neurosurgery. 2016; 125: 759-65.

2. Okamoto Y, Okamoto F, Yamada S, et al. Vision-related quality of life after transsphenoidal surgery for pituitary adenoma. Investigative ophthalmology \& visual science. 2010; 51: 3405-10.

3. Kerrison JB, Lynn MJ, Baer CA, et al. Stages of improvement in visual fields after pituitary tumor resection. American journal of ophthalmology. 2000; 130: 813-20.

4. Incesu AI, Sobaci G. Malingering or simulation in ophthalmology-visual acuity. International journal of ophthalmology. 2011; 4: 558-66.

5. Fox MD, Raichle ME. Spontaneous fluctuations in brain activity observed with functional magnetic resonance imaging. Nature reviews Neuroscience. 2007; 8: 700-11.

6. Raichle ME, Snyder AZ. A default mode of brain function: a brief history of an evolving idea. NeuroImage. 2007; 37: 1083-90; discussion 97-9.

7. Tomasi D, Wang R, Wang GJ, et al. Functional connectivity and brain activation: a synergistic approach. Cerebral cortex. 2014; 24: 2619-29.

8. Lewis CM, Baldassarre A, Committeri $\mathrm{G}$, et al. Learning sculpts the spontaneous activity of the resting human brain. Proceedings of the National Academy of Sciences of the United States of America. 2009; 106: 17558-63.

9. Deshpande G, LaConte S, Peltier S, et al. Integrated local correlation: a new measure of local coherence in fMRI data. Human brain mapping. 2009; 30: 13-23.

10. Wang J, Wei Q, Yuan X, et al. Local functional connectivity density is closely associated with the response of electroconvulsive therapy in major depressive disorder. Journal of affective disorders. 2018; 225: 658-64.

11. Huber E, Webster JM, Brewer AA, et al. A lack of experience-dependent plasticity after more than a decade of recovered sight. Psychological science. 2015; 26: 393-401.

12. Korsholm $\mathrm{K}$, Madsen $\mathrm{KH}$, Frederiksen JL, et al. Cortical neuroplasticity in patients recovering from acute optic neuritis. NeuroImage. 2008; 42: 836-44

13. Korsholm K, Madsen KH, Frederiksen JL, et al. Recovery from optic neuritis: an ROI-based analysis of LGN and visual cortical areas. Brain : a journal of neurology. 2007; 130: 1244-53.

14. Jenkins TM, Toosy AT, Ciccarelli O, et al. Neuroplasticity predicts outcome of optic neuritis independent of tissue damage. Annals of neurology. 2010; 67: 99-113.

15. Dilks DD, Serences JT, Rosenau BJ, et al. Human adult cortical reorganization and consequent visual distortion. The Journal of neuroscience : the official journal of the Society for Neuroscience. 2007; 27: 9585-94.

16. Chouinard PA, Striemer CL, Ryu WH, et al. Retinotopic organization of the visual cortex before and after decompression of the optic chiasm in a patient with pituitary macroadenoma. Journal of neurosurgery. 2012; 117: 218-24.

17. Paul DA, Gaffin-Cahn E, Hintz EB, et al. White matter changes linked to visual recovery after nerve decompression. Science translational medicine. 2014; 6: 266ra173.

18. Song G, Qiu J, Li C, et al. Alterations of regional homogeneity and functional connectivity in pituitary adenoma patients with visual impairment. Scientific reports. 2017; 7: 13074

19. Qian HY, Wang XC, Wang ZY, et al. Changes in the Vision-related Resting-state Network in Pituitary Adenoma Patients After Vision Improvement. Chinese medical journal. 2015; 128: 1171-6.

20. Qian H, Wang X, Wang Z, et al. Altered Vision-Related Resting-State Activity in Pituitary Adenoma Patients with Visual Damage. PloS one. 2016; 11: e0160119.

21. Fahlbusch R, Schott $\mathrm{W}$. Pterional surgery of meningiomas of the tuberculum sellae and planum sphenoidale: surgical results with special consideration of ophthalmological and endocrinological outcomes. Journal of neurosurgery. 2002; 96: 235-43.

22. Metwali $H$, Giordano $M$, Kniese $K$, et al. Prognostic significance of intraoperative change in the fractional anisotropy and the volume of the optic chiasma during resection of suprasellar tumors. Journal of neurosurgery. 2018; 128: $1479-85$.

23. Glasser MF, Sotiropoulos SN, Wilson JA, et al. The minimal preprocessing pipelines for the Human Connectome Project. NeuroImage. 2013; 80: 105-24.

24. Whitfield-Gabrieli S, Nieto-Castanon A. Conn: a functional connectivity toolbox for correlated and anticorrelated brain networks. Brain connectivity. 2012; 2: 125-41.

25. Creem SH, Proffitt DR. Defining the cortical visual systems: "what", "where", and "how". Acta psychologica. 2001; 107: 43-68.

26. Hasnain MK, Fox PT, Woldorff MG. Intersubject variability of functional areas in the human visual cortex. Human brain mapping. 1998; 6: 301-15.

27. Scholl B, Burge J, Priebe NJ. Binocular integration and disparity selectivity in mouse primary visual cortex. Journal of neurophysiology. 2013; 109: 3013-24.

28. Luomaranta T, Raappana A, Saarela V, et al. Factors Affecting the Visual Outcome of Pituitary Adenoma Patients Treated with Endoscopic Transsphenoidal Surgery. World neurosurgery. 2017; 105: 422-31.

29. Muskens IS, Zamanipoor Najafabadi AH, Briceno V, et al. Visual outcomes after endoscopic endonasal pituitary adenoma resection: a systematic review and meta-analysis. Pituitary. 2017; 20: 539-52.
30. Campbell PG, McGettigan B, Luginbuhl A, et al. Endocrinological and ophthalmological consequences of an initial endonasal endoscopic approach for resection of craniopharyngiomas. Neurosurgical focus. 2010; 28: E8.

31. Seuk JW, Kim CH, Yang MS, et al. Visual outcome after transsphenoidal surgery in patients with pituitary apoplexy. Journal of Korean Neurosurgical Society. 2011; 49: 339-44.

32. Juraschka $\mathrm{K}$, Khan $\mathrm{OH}$, Godoy $\mathrm{BL}$, et al. Endoscopic endonasal transsphenoidal approach to large and giant pituitary adenomas: institutional experience and predictors of extent of resection. Journal of neurosurgery. 2014; 121: $75-83$

33. Woo HJ, Hwang JH, Hwang SK, et al. Clinical outcome of cranial neuropathy in patients with pituitary apoplexy. Journal of Korean Neurosurgical Society. 2010; 48: 213-8.

34. Bullmore E, Sporns O. The economy of brain network organization. Nature reviews Neuroscience. 2012; 13: 336-49.

35. Buckner RL, Vincent JL. Unrest at rest: default activity and spontaneous network correlations. NeuroImage. 2007; 37: 1091-6; discussion 7-9.

36. Kelly C, Castellanos FX. Strengthening connections: functional connectivity and brain plasticity. Neuropsychology review. 2014; 24: 63-76.

37. Furmanski CS, Schluppeck D, Engel SA. Learning strengthens the response of primary visual cortex to simple patterns. Current biology : CB. 2004; 14: 573-8.

38. Schwartz S, Maquet P, Frith C. Neural correlates of perceptual learning: a functional MRI study of visual texture discrimination. Proceedings of the National Academy of Sciences of the United States of America. 2002; 99: 17137-42.

39. Yotsumoto Y, Watanabe T, Sasaki Y. Different dynamics of performance and brain activation in the time course of perceptual learning. Neuron. 2008; 57: 827-33.

40. Kang DW, Kim D, Chang LH, et al. Structural and Functional Connectivity Changes Beyond Visual Cortex in a Later Phase of Visual Perceptual Learning. Scientific reports. 2018; 8: 5186.

41. Hillary FG, Roman CA, Venkatesan U, et al. Hyperconnectivity is a fundamental response to neurological disruption. Neuropsychology. 2015; 29: 59-75.

42. Jia X, Ma S, Jiang S, et al. Disrupted Coupling Between the Spontaneous Fluctuation and Functional Connectivity in Idiopathic Generalized Epilepsy. Frontiers in neurology. 2018; 9: 838

43. Konstantinou N, Pettemeridou E, Stamatakis EA, et al. Altered Resting Functional Connectivity Is Related to Cognitive Outcome in Males With Moderate-Severe Traumatic Brain Injury. Frontiers in neurology. 2018; 9: 1163.

44. Ortner $\mathrm{M}$, Pasquini $\mathrm{L}_{\text {, Barat }} \mathrm{M}$, et al Progressively Disrupted Intrinsic Functional Connectivity of Basolateral Amygdala in Very Early Alzheimer's Disease. Frontiers in neurology. 2016; 7: 132 .

45. Watson JD, Myers R, Frackowiak RS, et al. Area V5 of the human brain: evidence from a combined study using positron emission tomography and magnetic resonance imaging. Cerebral cortex. 1993; 3: 79-94.

46. Amano K, Wandell BA, Dumoulin SO. Visual field maps, population receptive field sizes, and visual field coverage in the human MT+ complex. Journal of neurophysiology. 2009; 102: 2704-18.

47. Kaas A, Weigelt S, Roebroeck A, et al. Imagery of a moving object: the role of occipital cortex and human MT/V5+. NeuroImage. 2010; 49: 794-804

48. Johnson-Frey SH, Newman-Norlund R, Grafton ST. A distributed left hemisphere network active during planning of everyday tool use skills. Cerebral cortex. 2005; 15: 681-95.

49. Carota F, Posada A, Harquel S, et al. Neural dynamics of the intention to speak. Cerebral cortex. 2010; 20: 1891-7.

50. Kringelbach ML. The human orbitofrontal cortex: linking reward to hedonic experience. Nature reviews Neuroscience. 2005; 6: 691-702. 